\title{
Implementation of Stereo Vision Semi-Global Block Matching Methods for Distance Measurement
}

\author{
Raden Arief Setyawan ${ }^{1}$, Rudy Sunoko ${ }^{2}$, Mochammad Agus Choiron ${ }^{3}$, Panca Mudji Rahardjo ${ }^{4}$ \\ ${ }^{1,4}$ Electrical Engineering Department, Brawijaya University, Indonesia \\ ${ }^{2,3}$ Mechanical Engineering Department, Brawijaya University, Indonesia
}

\section{Article Info \\ Article history: \\ Received May 9, 2018 \\ Revised Jul 12, 2018 \\ Accepted Jul 21, 2018}

Keywords:

Distance Measurement

Stereo Matching

Stereo Vision

\begin{abstract}
Stereo vision has become an attractive topic research in the last decades. Many implementations such as the autonomous car, 3D movie, 3D object generation, are produced using this technique. The advantages of using two cameras in stereo vision are the disparity map between images. Disparity map will produce distance estimation of the object. Distance measurement is a crucial parameter for an autonomous car. The distance between corresponding points between the left and right images must be precisely measured to get an accurate distance. One of the most challenging in stereo vision is to find corresponding points between left and right images (stereo matching). This paper proposed distance measurement using stereo vision using Semi-Global Block Matching algorithm for stereo matching purpose. The object is captured using a calibrated stereo camera. The images pair then optimized using WLS Filter to reduce noises. The implementation results of this algorithm are furthermore converted to a metric unit for distance measurement. The result shows that the stereo vision distance measurement using Semi-Global Block Matching gives a good result. The obtained best result of this work contains error of less than $1 \%$ for $1 \mathrm{~m}$ distance
\end{abstract}

Copyright (C) 2018 Institute of Advanced Engineering and Science. All rights reserved.

\section{Corresponding Author:}

Raden Arief Setyawan,

Electrical Engineering Department, Brawijaya University, Indonesia.

Email: rarief@ub.ac.id

\section{INTRODUCTION}

The development of digital image technology nowadays is growing rapidly. Higher camera quality, faster image processing technology, and image processing methods give a significant impact to the daily human life. One of the most attractive research in digital image technology is Stereo Vision/3D imaging. Stereo vision is a method to form 3-dimensional images using two cameras. This method will generate a depth map which provides information about the distance between an object and the camera. However, the distance obtained in this measurement is still limited to the assumption of the object's proximity. Distance measurement is essential in various applications, such as robotics, autonomous car, and so forth. Several previous studies have attempted to measure distance using stereo vision. Tjandranegara et al. offer a method for estimating the distance between objects by using two cameras [1]. The method offered is pure of mathematical calculations without implementation. Mrovlje et al. attempted to apply distance measurements to measure objects with distances between $10 \mathrm{~m}-60 \mathrm{~m}$ [2]. Mahammed, in another study, was comparing stereo vision measurements with a digital laser range-finder [3]. The measurement of disparity has been done utilizing the Fast Matching using Cross-Correlation Based Sum-of-Squared Differences method. This research gives the relative measurement error between $1.13 \%$ and $2.05 \%$.

Based on the previous work, one of the most important steps to maintain the accuracy of measurement is the stereo matching method. Stereo matching is a method to find corresponding points in a pair of images. Corresponding points can be recognized through several efficient matching 
algorithms [4], [5]. The quality of stereo matching related to measurement accuracy. Semi-Global Matching, initially proposed by Hirschmuller, has proven as a powerful stereo matching algorithm for various application. This method minimizes a global cost function in one dimension.

This study aims to test the accuracy of distance measurement using stereo vision with SGBM Algorithm in stereo matching process. In this work, two Logitech web cameras prepared as the stereo camera. The Camera calibration is a critical step to get epipolar lines between images pair. It must be done before the measurement process takes places. Pair of images captured by the camera is processed using Stereo Global Block Matching (SGBM). SGBM is a modified version of the Semi-Global Matching (SBM) algorithm proposed by Hirschmuller [6], [7]. The implementation of this method using the OpenCV Library [8]. Next, the WLS Filter used to optimize the output of stereo matching [9]. Measurement calibration needed to convert from pixel distance to metrics unit. Subsequently, the obtained data of the pixel distance are converted into metrics unit. The calibration result is calculated to find the regression formula. This formula then applied in the conversion of the disparity value to the metric unit. This system tested with another object and distance variation to find the measurement accuracy.

\section{STEREO VISION}

Charles Wheatstone initiated the research on stereo vision / stereoscopic measurement in 1838 by inventing stereo images using equipment to view them. Two left, and right cameras are used to mimic the human eye and placed at a certain distance [2]. Assuming that both cameras are perfectly aligned, two images from the left and right cameras are captured with different angles. Furthermore, both images are used to calculate the distance between objects with the camera using trigonometric principles.

Figure 1 describes the basic principle of distance measurement. As it indicates, $\mathrm{C} 1$ represents the left camera, and C2 represents the right camera. The Base (B) represents the distance between the two cameras. $\mathrm{D}$ is the distance between the Object and the cameras. The angle $\varphi 0$ is the view angle of the camera. The angles $\varphi 1$ and $\varphi 2$ are the angles between the normal view and the object locations.

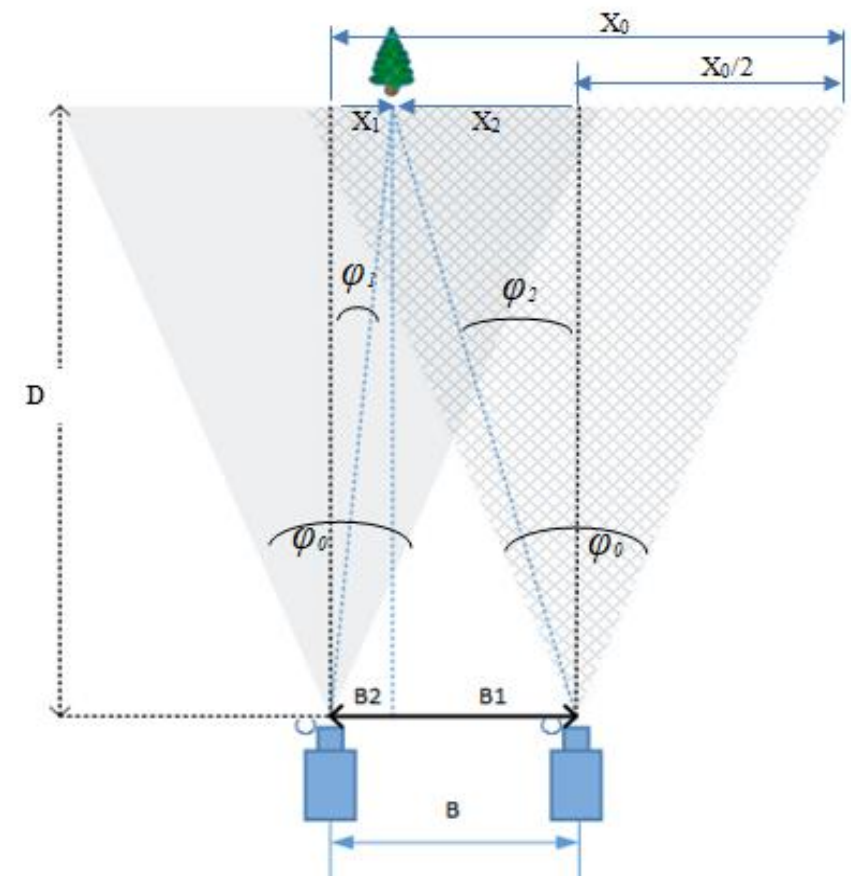

Figure 1. The Stereo Vision layout

The Distance (D) can be calculated using the basic trigonometry as follows.

$$
D=\frac{B}{\tan \varphi_{1}+\tan \varphi_{2}}
$$




$$
D=\frac{B X_{0}}{2 \tan \left(\frac{\varphi_{0}}{2}\right)\left(X_{L}-X_{D}\right)}
$$

The Distance (D) can be found out using Equation 2 if the distance between the cameras (B), the view angle of the camera $(\varphi 0)$ and the disparity of the object from the left and the right cameras are known. The result is expressed in pixels. Converting the pixel number into metrics unit requires a reference calibration.

\section{SEMI-GLOBAL BLOCK MATCHING AND WLS FILTER}

Semi-Global matching method objective is to minimize the cost function of Global matching method. Equation 3 describe the global cost function [7]

$$
E(D)=\sum_{P}\left(C(p, D p)+\sum_{q \in N p} P_{1} T|| D p-D q|=1|+\sum_{q \in N p} P_{2} T|| D p-D q|>1|\right)
$$

$\mathrm{C}(\mathrm{p}, \mathrm{Dp})$ is the sum of a pixel-wise matching cost for pixel $\mathrm{p}$ at their disparity Dp. The Function $\mathrm{T}$ will result in 1 if its argument is true, and 0 if false. $\mathrm{Np}$ is the neighboring pixel of p with the cost P1. P2 is adapted to the local intensity gradient for the neighboring pixel p and q. SGM calculates E(D) along with the 1D path from 8 directions toward each pixel of interest (Figure 2).

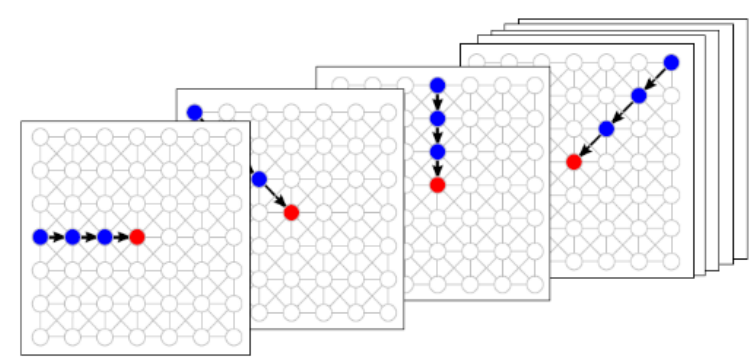

Figure 2. Path Direction of SGM

The Cost of all path is summed for each pixel and disparity. The disparity is then determined by winner-takes-All principle. The implementation of the SGM method in this work utilized SGBM library of OpenCV [10]. SGBM is a modified version of the SGM Method with the additional parameter to limit disparity value, windows size and uniqueness ratio. The next step is the noises minimization by using the Weighted Least Squares filter [9].

\section{EXPERIMENT}

The experiment procedure being performed on this work is described in Figure 3. The stereo images are produced using two Logitech 920 USB webcams. The distance between the two cameras is $10 \mathrm{~cm}$. Based on the trigonometry calculation principle, the range of distance measurement is related to the distance between the two stereo cameras. With $10 \mathrm{~cm}$ of distance, the expected measurement range is in between $50 \mathrm{~cm}$ to $180 \mathrm{~cm}$. The view angle of this webcam is 78 degrees. This wide angle increases the possibility of image distortion; therefore, the camera calibration is essential.

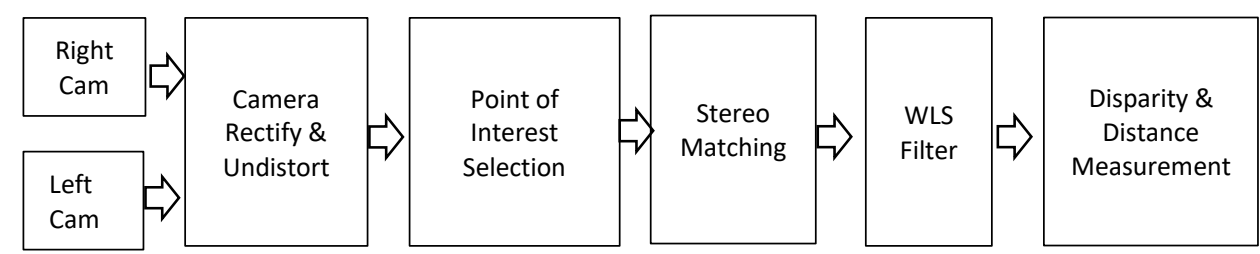

Figure 3. The distance measurement procedure 
The camera calibration is required to ensure that both cameras are in perfect parallel position and to remove distortion [11]. Camera calibration also generates the epipolar lines between corresponding points on images pair. These lines change the complexity of searching from 2D to 1D, resulting in less searching time. The calibration is performed using the standard chess-board picture. Figure 4 and Figure 5 displays the image calibration process. The following step is the detection of the object. The object is designed using a specific pattern to reduce the finding problem.

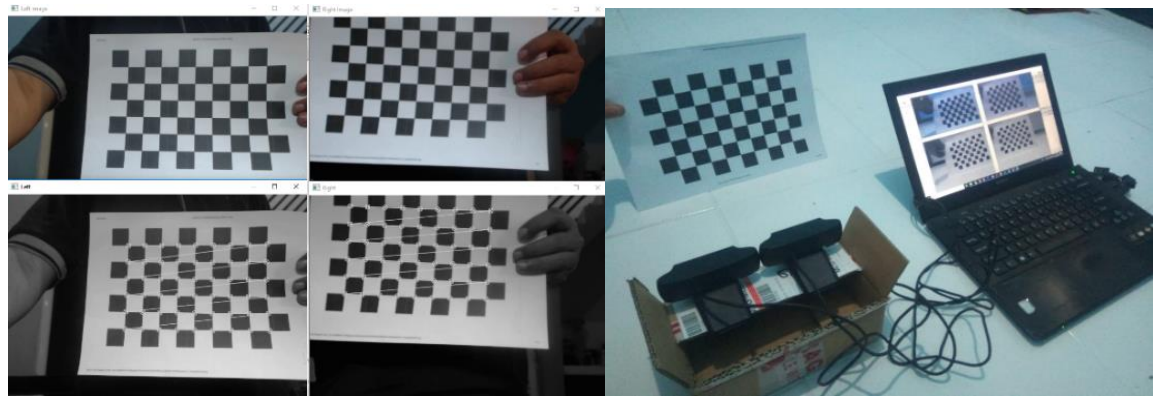

Figure 4. The camera calibration

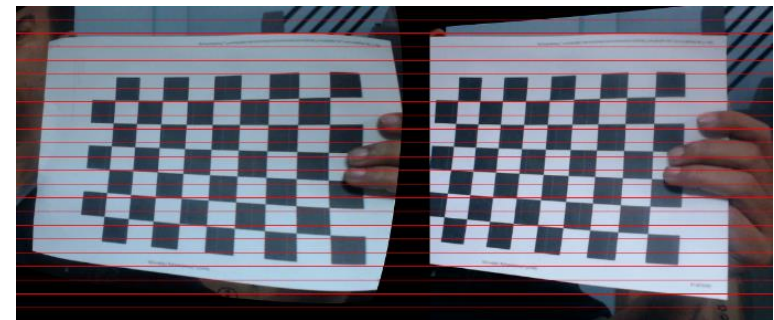

Figure 5. The Epipolar Lines on the calibrated camera

After the preparation of both cameras, they are ready to measure the distance of the object. The calibration matrix produced by the calibration process is required to remove camera distortion and to find the epipolar lines on both images. The following step is the stereo calculation process to find the disparity map. The Semi-Global Block Matching Algorithm (SGBM) is used for this purpose.

The stereo matching algorithm is an essential step in generating the disparity map. In this experiment, the object is positioned from $50 \mathrm{~cm}$ to $180 \mathrm{~cm}$ in front of the camera. Figure 6 and Figure 7 shows the procedure of the experiment.

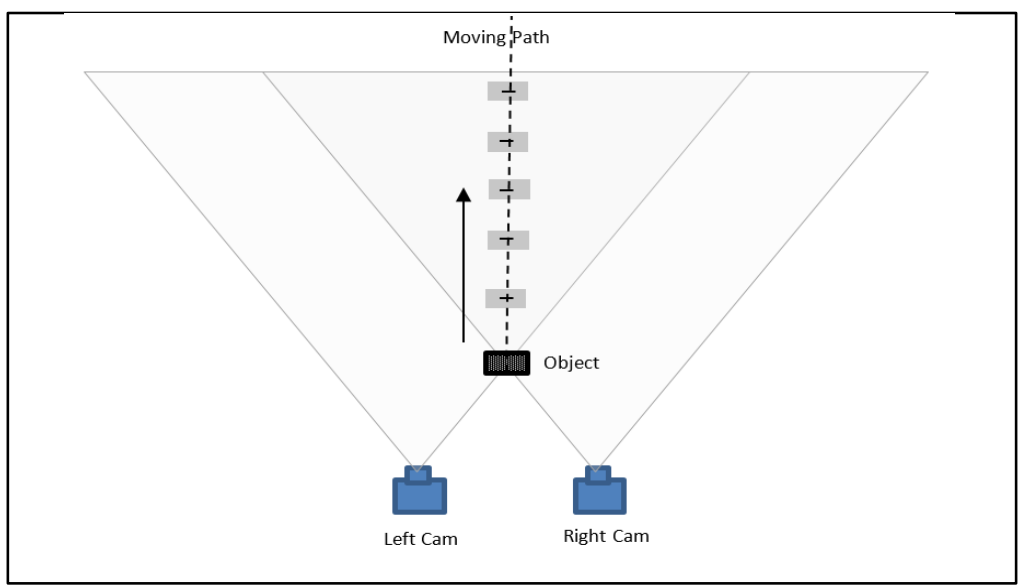

Figure 6. Experiment Procedure 
For the first setup, an object is placed in $50 \mathrm{~cm}$ in front of the camera. A ruler is placed on the path between the two cameras as a reference. The measurement process is executed by double-clicking the selected object on the screen. The output of measurement is written on a text file. The next step is done by increasing the distance between the camera and the object with an interval of $10 \mathrm{~cm}$. The result of the measurement algorithm is expressed in pixels value.
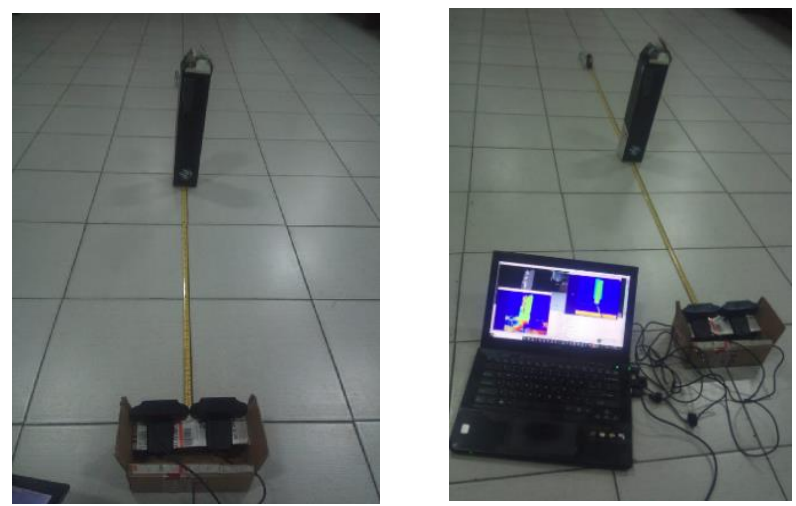

Figure 7. Distance Measurement Eksperiment

\section{RESULTS AND ANALYSIS}

The first experiment is to determine the relationship between the disparity and the object distance. Table 1 shows the relationship between the object distances with the disparity between images pair. The disparity value is the average or $3 \times 3$ pixel around the point of interest (POI). Figure 8 indicates that the correlation between the distance and the average disparity is not linear. The disparity value increases exponentially when the object becomes closer to the camera, and slowly decreases when the distance increases. Using the regression method the polynomial equation of the chart can be found and can be used as the conversion formula.

Table 1. Reference Distance vs

\begin{tabular}{lr}
\multicolumn{2}{c}{ Average Disparity Value } \\
\hline $\begin{array}{c}\text { Reference } \\
\text { Distance }(\mathbf{c m})\end{array}$ & $\begin{array}{c}\text { Average of 3x3 } \\
\text { Disparity value }\end{array}$ \\
\hline 50 & 0.988462095 \\
60 & 0.85396412 \\
70 & 0.717343027 \\
80 & 0.616258409 \\
90 & 0.539270472 \\
100 & 0.479804145 \\
110 & 0.430902778 \\
120 & 0.391988571 \\
130 & 0.358893953 \\
140 & 0.332242839 \\
150 & 0.306213831 \\
160 & 0.287497496 \\
170 & 0.249784736 \\
180 & 0.232408312 \\
\hline
\end{tabular}

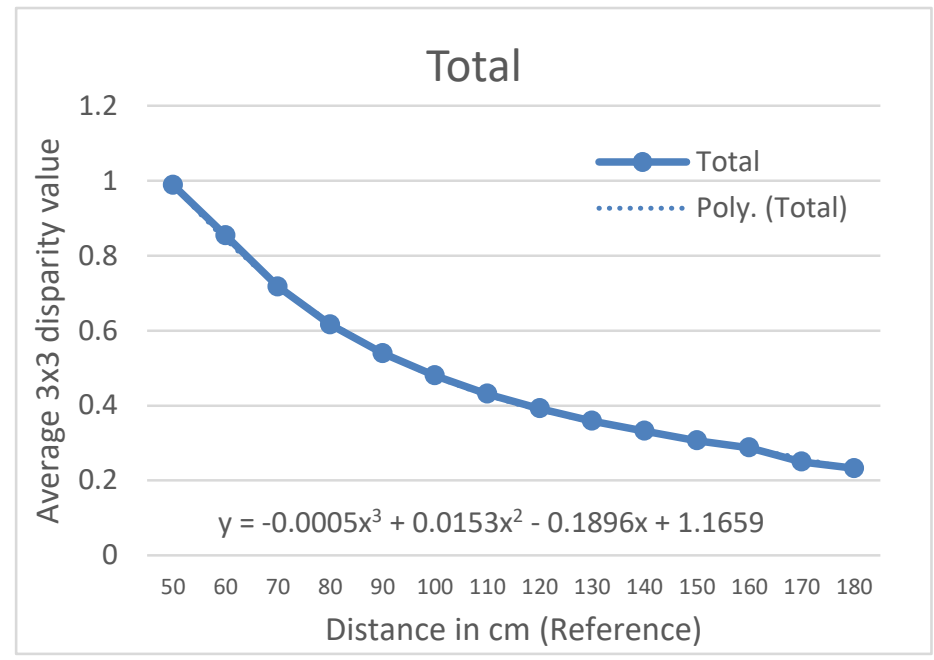

Figure 8. Distace vs disparity

The effect of the WLS filter implementation in this work is shown in Figure 9. It can be seen that without the WLS filtering the output image is very noisy. 

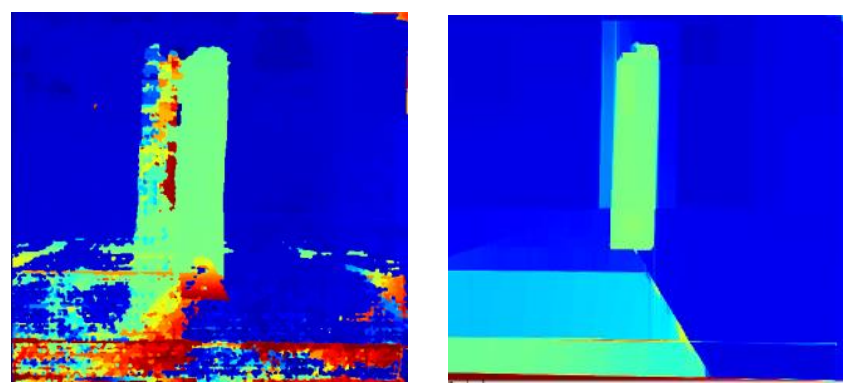

Figure 9. Result of WLS Filter Implementation. Left image, before WLS filter, right image after WLS Filter

The second experiment is to determine the measurement error. Table 2 shows the result of the measurement. The obtained error rate was very high when the object was too close to the camera. The distance between the two cameras is $10 \mathrm{~cm}$, giving the best measurement accuracy on $100 \mathrm{~cm}$. When the distance between the camera and the object increases, the error measurement will increase. The highest error has been achieved when the reference distance was $50 \mathrm{~cm}$ with $2.5 \%$ error. If the object distance is less than $100 \mathrm{~cm}$, the result of stereo vision measurement is higher than reference, but when the distance of object is more than $100 \mathrm{~cm}$, the result of measurement is less than the reference.

Table 2. Measurement results

\begin{tabular}{ccccccc}
\hline \multirow{2}{*}{ Distance Ref $(\mathrm{cm})$} & \multicolumn{9}{c}{ Stere vision measurement } & \multirow{2}{*}{ Error } \\
\cline { 2 - 5 } & 1 & 2 & 3 & 4 & Avg & \\
\hline 50 & 51 & 50 & 51 & 53 & 51.25 & $2.50 \%$ \\
60 & 61 & 61 & 62 & 61 & 61.25 & $2.08 \%$ \\
70 & 70 & 75 & 71 & 69 & 71.25 & $1.79 \%$ \\
80 & 83 & 80 & 82 & 79 & 81 & $1.25 \%$ \\
90 & 90 & 92 & 93 & 89 & 91 & $1.11 \%$ \\
100 & 101 & 102 & 99 & 99 & 100.25 & $0.25 \%$ \\
110 & 107 & 109 & 114 & 113 & 110.75 & $0.68 \%$ \\
120 & 116 & 119 & 121 & 119 & 118.75 & $1.04 \%$ \\
130 & 129 & 128 & 129 & 128 & 128.5 & $1.15 \%$ \\
140 & 130 & 142 & 141 & 140 & 138.25 & $1.25 \%$ \\
150 & 149 & 148 & 147 & 148 & 148 & $1.33 \%$ \\
160 & 158 & 159 & 159 & 154 & 157.5 & $1.56 \%$ \\
170 & 169 & 168 & 167 & 165 & 167.25 & $1.62 \%$ \\
180 & 177 & 179 & 175 & 176 & 176.75 & $1.81 \%$ \\
\hline
\end{tabular}

Figure 10 shows that the measurement result is higher than reference when the distance between object and camera is below $1 \mathrm{~m}$ and lower if the distance is more than $1 \mathrm{~m}$.

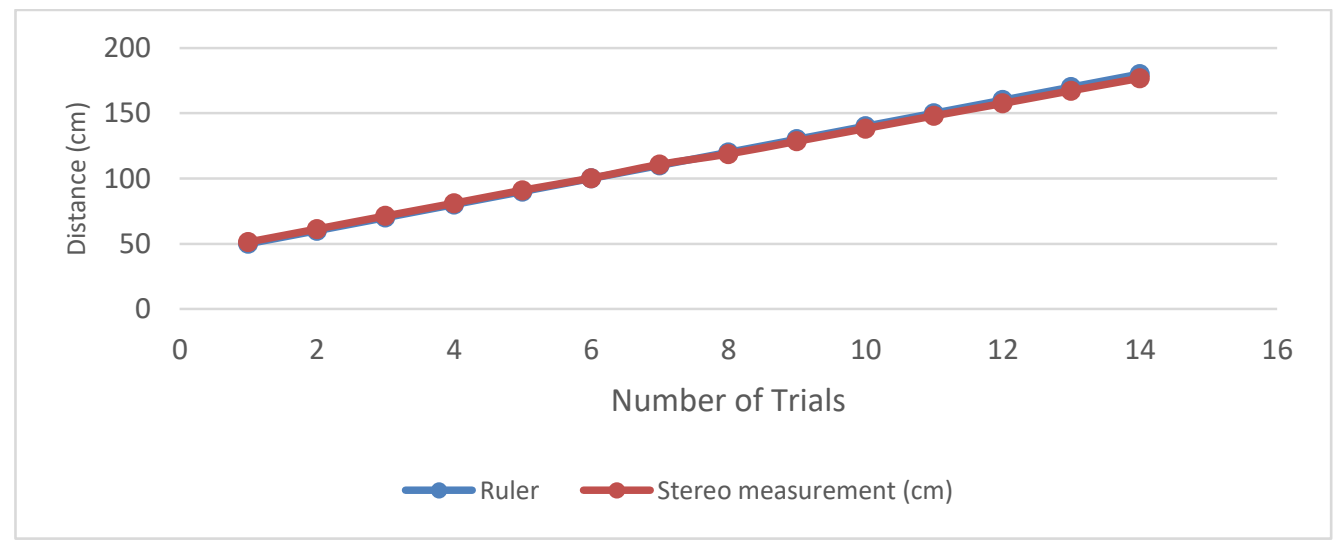

Figure 10. Measurement Using SGBM Method 


\section{CONCLUSION}

The distance measurement using stereo vision method has been proposed. The result of this work indicates that the Semi-Global Block Matching method gives a good result, with an error rate of less than $10 \%$, but the measurement result is not correlated linearly with the real measurement. It can be concluded that the stereo vision method provides an alternative for non-contact distance measurement. However, because of the complexity of supported components, very careful calibration and precise adjustment between cameras are highly required to give a better result.

\section{REFERENCES}

[1] E. Tjandranegara. "Distance Estimation Algorithm for Stereo Pair Images". Comput. Eng., 2005.

[2] J. Mrovlje and D. Vran. "Distance measuring based on stereoscopic pictures" 9th International PhD Workshop on System Control Young Generation Viewpoint. 2008;2:1-6.

[3] M. a Mahammed, A. I. Melhum, and F. a Kochery, "Object Distance Measurement by Stereo Vision," International Journal of Science and Applied Information Technology. 2013;2(2): 5-8.

[4] D. Scharstein, R. Szeliski, and R. Zabih, "A taxonomy and evaluation of dense two-frame stereo correspondence algorithms,” International Journal of Computer Vision. 2002, 47(1): 131-140.

[5] G. Cocorullo, P. Corsonello, F. Frustaci, and S. Perri, "An efficient hardware-oriented stereo matching algorithm," Microprocessors and Microsystems. 2016; 46: 21-33.

[6] H. Hirschuller, "Stereo processing by semi-gobal matching and mutual information," IEEE Trans Pattern Anal Mach Intell. 2008; 30(2): 328-341.

[7] H. Hirschmüller and D. Scharstein, "Evaluation of Cost Functions for Stereo Matching," 2007 IEEE Conference Computer Vision and Pattern Recognition. 2007: 1-8.

[8] U. Stephane, VUJASINOVIC Frederic, "Stereo-Vision," GitHub, 2018 Available: https://github.com/LearnTechWithUs/Stereo-Vision.

[9] W. Liu, X. Chen, C. Shen, Z. Liu, and J. Yang, "Semi-Global Weighted Least Squares in Image Filtering," Proc. IEEE International Conference on Computer Vision, 2017;5862-5870.

[10] C. Lü, X. Wang, and Y. Shen, "A stereo vision measurement system Based on OpenCV," Proceedings of the 2013 6th International Congress on Image and Signal Processing. 2013, 2:718-722.

[11] W. Sankowski, M. Włodarczyk, D. Kacperski, and K. Grabowski, "Estimation of measurement uncertainty in stereo vision system \&,” Image Vision Computing. 2017; 61:70-81. 